\title{
Establishing a deanery teaching programme for trainees sitting the MRCPCH clinical examination
}

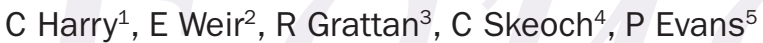

Abstract in the UK must obtain to progress with their specialty training. In August 2009 a structured clinical teaching programme was initiated in the West of Scotland deanery to provide teaching in the clinical examination curriculum, the structure and examination standards.

Design The training programme runs three times a year, commencing two months prior to each exam. Trainees are sent questionnaires to enable improvement of the programme.

Results A total of 127 trainees have participated in the programme over five years (August 2009-2014). Trainees report strengths of the programme include communication teaching $(n=14)$ and general teaching $(n=11)$. Challenges encountered include difficulties attending teaching sessions due to clinical commitments $(n=5)$.

Conclusions Future development will focus on creating a robust system enabling this programme to continue and establishment of a key group of paediatric consultants, who can provide consistent teaching reflecting the realities of the examination.

Keywords: curriculum, continuing education, graduate education, paediatrics, professional competence, teaching

Declaration of interests: No conflict of interests declared

\section{Correspondence to:}

C Harry

Paediatric Intensive Care

Unit

Royal Hospital for Children 1345 Govan Road Glasgow G51 4TF UK

Email: christina_harry@hotmail.com

\section{Background}

Membership of the Royal College of Paediatrics and Child Health (MRCPCH) is an essential qualification all paediatric trainees in the UK must obtain to progress past specialty training year three. The MRCPCH clinical examination is the final part required for this qualification. Prior to 2009 there had not been a formalised training programme for trainees in the West of Scotland deanery preparing for this examination.

The aim of the examination is to assess whether candidates have reached the standard in clinical skills expected of a newly appointed Specialist Registrar. ${ }^{1}$ Candidates are expected to demonstrated proficiency in:

- Communication

- History taking and management planning

- Establishing rapport with parents and children

- Physical examination

- Child development

- Clinical judgement

- Organisation of thoughts and actions

- Recognition of acute illness
- Knowledge of paediatrics and child health

- Professional behaviour

- Ethical practice

The MRCPCH clinical examination (held three times a year, in February, June and October) follows an objective structured clinical examination (OSCE) style format. ${ }^{2}$ Stations are set to test candidates in:

- Child development

- Communication skills

- History-taking and management planning

- Recognition and diagnosis of clinical signs and symptoms

- Physical examination skills

\section{Objectives}

Prior to 2009, candidate preparation was based on ad hoc tutorials and teaching sessions organised by paediatric trainees sitting the examination but with no advice on the structure, expectations or marking of the examination. In 2009, a small working group was established with the 
aim of organising structured teaching and support for the paediatric trainees sitting the MRCPCH clinical examination. This programme was evaluated in the form of trainee questionnaires and the feedback reviewed, enabling the group to modify the process for future trainee groups to develop a comprehensive and progressive training package. ${ }^{3}$

\section{Methods}

\section{Organisation of the teaching programme}

The programme was initially organised by three individuals in the West of Scotland; a senior paediatric specialty trainee, a consultant neonatologist with involvement in the $\mathrm{RCPCH}$ examination process and a Senior University Teacher in Medical Education (SUTME) at Glasgow University. Two years later, a further paediatric specialty trainee joined the group and another a year later. This group organised all teaching and administrative tasks so trainees did not have to pay any fees. The teaching sessions were run at a tertiary paediatric training hospital in Glasgow. Two months prior to the examination, all eligible trainees in the deanery were emailed inviting them to participate in the programme. These trainees were identified by obtaining a list of junior paediatric trainees (ST1-ST3) who had not yet obtained their $\mathrm{MRCPCH}$ and also trainees in paediatric non-training posts from the Paediatric Training Programme Director. Teaching sessions were organised with paediatric consultants. Eligible trainees were advised by email as to the details of these sessions. Teaching sessions typically lasted an hour. In addition, trainees were provided with a weekly update of teaching sessions for the coming week. While formulating the programme, three main points were addressed: (i) the curriculum for the teaching programme; (ii) the style of teaching; and (iii) evaluation of the programme.

\section{Curriculum}

There was an initial introduction session focusing on what was important in the examination and mapping the curriculum. In this session, expected standards and anchor statements were clarified, as stated on the RCPCH website. ${ }^{1}$ Anchor statements outlined the expected general standard for each station in the OSCE. They consist of a list of components which contribute to assessment of the candidate's performance and are provided to examiners to guide them with candidate assessment. This initial session enabled trainees to ask practical questions regarding the format of the examination. Trainees were able to seek advice regarding their concerns or questions they may have and encouraged to review the RCPCH website.

Clinical sessions were organised to begin two months before the examination. Some of these focused on history taking, clinical examination and communication skills. Other sessions focused on specialty areas of paediatrics and were delivered by specialists in these areas.

\section{Style of teaching}

In addition to the curriculum, it is important to provide trainees with assistance in the approach to the clinical examination. While advice on this topic was given in the introduction session, clinical sessions were structured with this in mind.

Clinical sessions highlighting a general approach to the clinical stations were organised. Anchor statements were used to encourage small groups of candidates to recognise and provide constructive feedback using the same criteria as is used in the clinical examination.

Structured teaching was provided for subspecialty paediatrics covered in the exam. Specialist paediatric consultants gave teaching in their area of expertise, in particular covering correct examination techniques and the standard expected of trainees in that subspecialty in the exam.

To supplement the structured teaching programme, trainees were advised of other opportunities in which they were able to consolidate their learning and preparation outwith organised clinical teaching. These include attendance at outpatient clinics, organising tutorial groups and approaching other paediatric consultants, registrars and health practitioners for further teaching.

\section{Evaluation}

Following completion of the programme and MRCPCH clinical examination, there was an optional evaluation in the form of a structured questionnaire for the trainees. ${ }^{4}$ All trainees were sent a survey, consisting of 40 items on a 5-point Likert scale with invitations for open comments. Trainees then forwarded their completed questionnaire to a third party, who anonymously forwarded the results. This feedback allowed modification and subsequent improvement of the teaching programme. Results were collated over a five year period (October 2009-2014).

\section{Results}

The teaching programme commenced in August 2009 in time for the examination in October 2009. Surveys were sent to trainees who participated in the programme after their exam for a five year period. The attendance at these sessions and the survey response rate is shown in Figure 1.

The teaching programme was reported to be a useful and constructive experience. Specific comments generated from the free text section of the survey outlined that the more effective parts were the communication skills teaching and general teaching (Figure 2).

Trainees reported that the biggest challenge they encountered was their inability to attend teaching due to busy clinical workloads. Other concerns were that there were too few sessions for each specialty and some teaching was poor in standard or not exam-related. When asked how the programme could be improved, trainees felt the main points were providing more sessions (both generally and also for specialty teaching) and organising of a practice OSCE prior to the examination (Figures 3 and 4). 
Figure 1 Number of trainees involved in teaching programme and survey response rate

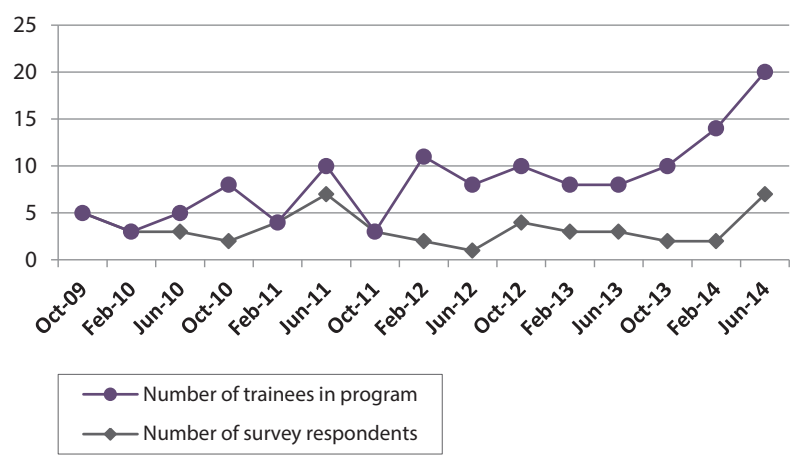

Figure 2 What was the most useful part of the programme?

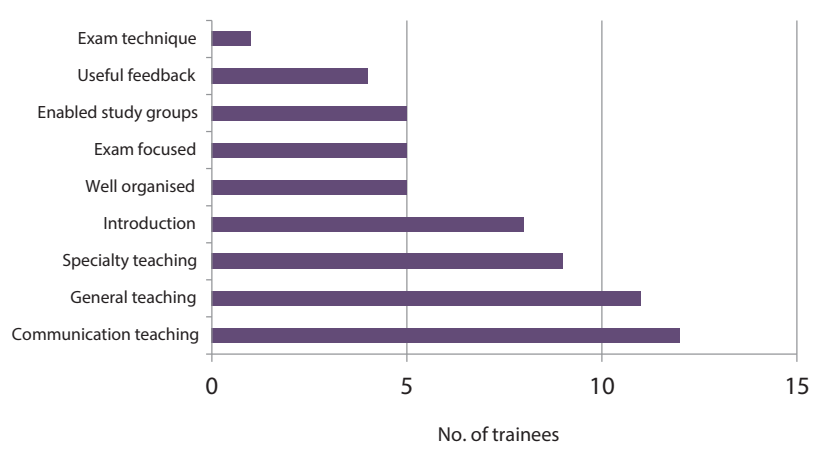

All respondents indicated that they felt an practice OSCE would be useful and that they would be willing to attend on a weekend for a practice OSCE prior to the examination.

\section{Discussion}

This teaching programme was implemented as it was acknowledged there was a lack of structured teaching for the $\mathrm{MRCPCH}$ clinical examination in Scotland. Prior to this, small groups of trainees often attempted to organise tutorials with paediatric consultants; however, there was no consistency or structure to this. There were no courses or teaching for this examination in Scotland, with candidates having to travel to London if they wished to attend an examination course; this was not covered in their study budget. A consultant with a senior examiner position was involved in elements regarding examination structure, curriculum and objectives. The SUTME was consulted and agreed to contribute and give advice on the educational components and feedback process. Trainees were encouraged to send their responses to the SUTME, who anonymised information for the group, and forwarded this on so that the points highlighted could be acted upon.

The programme has become a consistent addition to the West of Scotland paediatric training programme. In addition to teaching and feedback provided, it also gives opportunities to address the realities of the examination as well as form cohesive study groups. It is stated quite clearly in the literature provided that it provides core teaching
Figure $\mathbf{3}$ What was the least useful part of the programme?

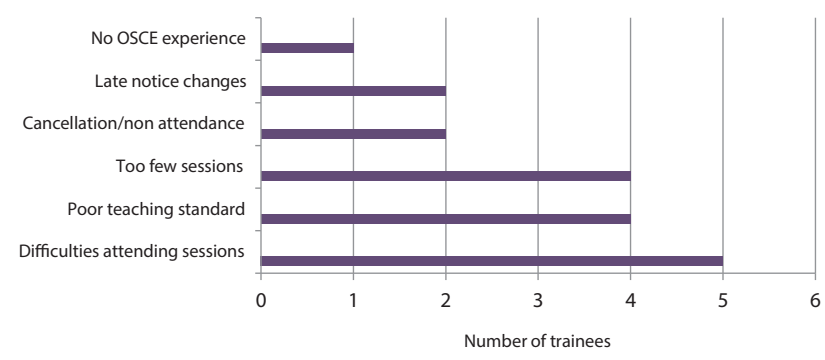

Figure 4 How could the programme be improved?

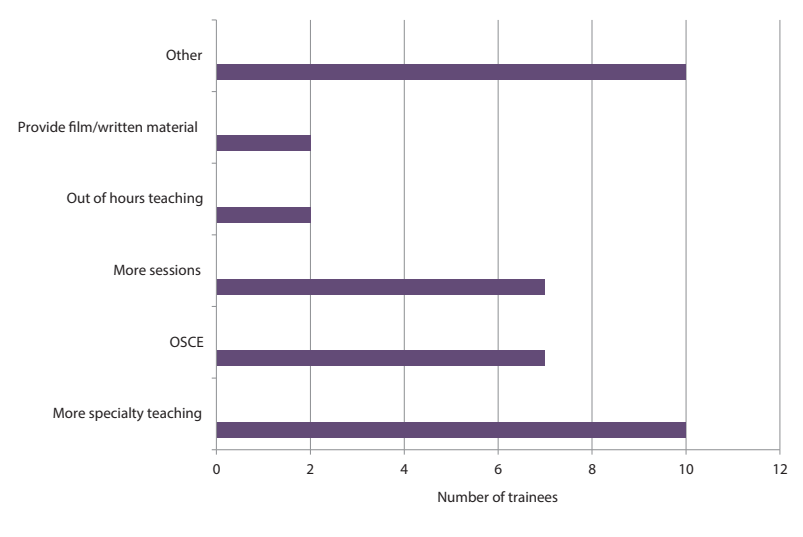

objectives but supplementary study and practice is required to pass the examination.

\section{Quality control of the programme}

It is essential that this programme aligns itself with the examination standards set out by the $\mathrm{RCPCH}$; the examination website clearly states that trainees should be able to perform at the level of a 'newly appointed registrar' (ST4). Therefore processes needed to be implemented to ensure the teaching delivered was appropriate and in line with the College recommendations so that candidates receive relevant and constructive teaching. This training programme sought to address the principles of the exam, practice of the exam process and individual specialty knowledge. It is vital that the components relating to the exam are taught by experienced $\mathrm{RCPCH}$ examiners. Similarly, it is essential that individuals teaching on paediatric subspecialties were well informed of the standard that the candidate is expected to perform at during the exam. Over time, we have tried to establish a core group of experienced individuals who are familiar with the exam syllabus with attempts to standardise teaching utilising the anchor statements provided by the RCPCH.

\section{Courses for MRCPCH clinical examination}

The availability of paediatric courses to prepare for the MRCPH clinical examination is varied throughout the UK. Many courses are commercially produced and expensive. Some are led by very experienced $\mathrm{RCPCH}$ clinical examiners and are designed primarily to familiarise the candidates with the principles of the exam. It is worth noting that $\mathrm{RCPCH}$ 
examiners should not provide service to commercial firms for their financial benefit. The RCPCH offers a two day Exam Skills Preparation Course in London, costing $£ 500 .^{5}$

The MRCPCH clinical examination is also run in Hong Kong, Singapore, Malaysia, India, Myanmar, Oman, Egypt, Saudi Arabia and the United Arab Emirates. ${ }^{6}$ Examinations are not necessarily offered at all of these centres at each sitting but there is the opportunity for trainees to apply to sit it. It is now standard practice that when the MRCPCH is introduced to a new region or country, there is examiner and candidate training prior to the first clinical exam. The content is lecture-based but mostly mock exam-based with extensive feedback and Q\&A sessions for candidates. The examiners are not paid for this but there is a small charge to the candidates to cover costs.

\section{Challenges and aspirations}

The most significant challenge to the programme is its longevity as it is run by a small nucleus of individuals with no administration support or funding and there are concerns that this project cannot be sustained. ${ }^{7}$ While the group is motivated for this to continue, it does not allow further development of the programme in response to the feedback provided by the trainees.

The most challenging aspect for trainees is that it is often difficult to attend teaching due to busy clinical commitments. Paediatric trainees, who are working elsewhere, especially at district general hospitals outside Glasgow, struggle to attend sessions and are thus disadvantaged. During busy clinical periods, both teachers and trainees struggle to attend some of the organised sessions and thus these are rescheduled or cancelled. Another common complaint relates to the quality of the teaching provided and insufficient number of sessions.

While attempts were made following this feedback to highlight these points to teachers and encourage review of the anchor statements, it is challenging with the current resources to implement change. Ideally, this would be an essential part of paediatric training. It would be beneficial to establish funding and administration support, so that this programme is sustainable and not dependent on a small group undertaking this in their own time. It would also be desirable to formulate a dedicated teaching faculty for this programme. The programme is indebted to many consultants and senior registrars who contribute enthusiastically and regularly to this process. We would like to establish a working group to ensure that standards are maintained to coincide with the evolution of the examination process, which would require the participation of individuals who are involved with the genesis, execution and quality assessment of the MRCPCH examination process in the UK. It would be necessary to embed the programme into the paediatric specialty programme so that bleep-free teaching could be organised.
This is difficult with current pressures on service provision and logistical challenges may dictate that some teaching be delivered out-of-hours. We would hope that development of a robust training programme and the reorientation of workplace attitudes in relation to the importance of examination training would facilitate this. While the group would not wish to profit from the programme, a small funding stream would be essential if aspirations of a practice OSCE were to be realised. For workload and service reasons, this would have to be run at the weekend and thus would require support from ancillary staff. The goodwill of parents and children would have to be further utilised to ensure adequate clinical cases. Organisation of an OSCE or mock exam is not a small undertaking and individual components of this are currently being explored by the organisers. ${ }^{8}$ Ideally, we would like to offer, along with Educational Supervisors, a mentorship programme to those trainees who struggle to pass the examination, ${ }^{9}$ so that problem areas can be addressed and optimise the opportunity of passing the clinical examination at the next sitting.

Doctors in training are an integral part of the NHS workforce, with one quarter of all doctors in the UK in training. ${ }^{10}$ Trainees' progression is dependent on successfully completing a number of examinations. It is essential to prepare trainees for these summative assessments. Such training should be embedded into specialty training programmes to provide essential support and education for trainees when a high clinical workload is the norm.

\section{Conclusions}

The initial steps in organising a structured teaching programme for paediatric trainees sitting their $\mathrm{MRCPCH}$ clinical examination have been taken with this programme now consolidated and in its eighth year. Review of the feedback has enabled modification of this programme to improve the process of preparation for future trainee groups. This programme provides essential support for paediatric trainees in their preparation for the clinical examination that is otherwise unavailable locally. Challenges encountered thus far relate mainly to difficulties attending teaching sessions due to clinical commitments, for both trainees and senior staff involved in teaching. Future development will focus on creating a robust system that will enable this programme to continue and establishment of a key group of paediatric consultants who can provide consistent teaching across the range of paediatric specialties in a way which reflects the realities of the clinical examination. The key points illustrated are transferrable and could be adopted by other specialist training providers outwith this specialty. Further studies investigating the training available for examinations in other medical specialties using a carefully constructed survey would allow for meaningful data collection in this area. (1) 


\section{References}

$1 \mathrm{RCPCH}$. Training, Examinations \& Professional Development. http:// www.rcpch.ac.uk/training-examinations (accessed 10/9/15).

2 Harden RM, Stevenson M, Downie WW et al. Assessment of clinical competence using objective structured examination. Brit Med J 1975; 1: 447-51.

3 Prideaux D. ABC of learning and teaching in medicine. Curriculum design. BMJ 2003; 326: 268-70.

4 Boynton PM. Hands-on guide to questionnaire research. Administering, analysing, and reporting your questionnaire. BM 2004; 328: 1372-5.

5 RCPCH. MRCPCH Clinical Exam Preparation Course (2 days). http:// www.rcpch.ac.uk/courses/mrcpch-clinical-exam-preparationcourse-2-days (accessed 31/1/17).

6 MRCPCH overseas clinical centres. http://www.rcpch.ac.uk/ system/files/protected/page/MRCPCH\%200verseas\%20Host\%20 contacts\%20-\%20(Updated\%2005.07.2016).pdf (accessed 31/1/17)
7 Loeser H, O'Sullivan P, Irby DM. Leadership lessons from curricular change at the University of California, San Francisco, School of Medicine. Acad Med 2007; 82: 324-30.

8 Skinner R, Wright CM, Craft AW. How to organize the paediatric MRCP (UK) part II clinical examination. Arch Dis Child 1997; 76: 545-8.

9 Sambuco D, Dabrowka A, DeCastro R et al. Negotiation in academic medicine: narratives of faculty researchers and their mentors. Acad Med 2013; 88: 505-11.

10 General Medical Council. The state of medical education and practice in the UK 2014. http://www.gmc-uk.org/SOMEP_2014 FINAL.pdf_58751753.pdf (accessed 31/1/17). 